\title{
Silicon Straight Tube Fluid Density Sensor
}

\author{
Mohammad Najmzadeh \\ Chalmers University of Technology \\ SE-412 96, Gothenburg, Sweden \\ Email: najmzade@student.chalmers.se
}

\author{
Sjoerd Haasl \\ Imego AB \\ SE-411 33, Gothenburg, Sweden
}

\author{
Peter Enoksson \\ Chalmers University of Technology \\ SE-412 96, Gothenburg, Sweden
}

\begin{abstract}
In this paper, a micromachined silicon straight tube is tested as a fluid density sensor. In comparison with other density measurement techniques, the use of micromachined tubes require small sample volumes and allows continuous monitoring of the fluid density in microfluidic systems. Different vibration modes of the sensor were detected and calibrated using a laser Doppler vibrometer (LDV).

Linearity, simplicity, the straightforward fabrication and evaluation, the low flow restriction and reduced risk of trapping gas in the sensor due the absence of corners are the design's main advantages. The ability of the sensor to measure density of multiphase fluids and provide accurate results independent of other fluid parameters, allows it to be used in varying fields such as the biomedical, pharmaceutical and petrochemical industries.
\end{abstract}

\section{INTRODUCTION}

A resonant densitometer is a sensor for measuring fluid density where a change in density results in a change in the mass loading of the resonator structure and thus a change in the resonance frequency. [1]

All the prior reported micromachined tube density sensors have corners [1]-[3]. The corners can add an additional pressure drop over the tube structure and also increase the risk of trapping micro particles and bubbles inside the tube leading to inaccuracy in the resonance frequency measurements [4]. Other microsystem techniques for measuring the density like using quartz tuning forks, vibrating microdiaphragms, membranes, cantilevers and magnetoelastic thin or thick films have been presented [5]-[10]. The advantages of using micromachined tubes lie in the low sample volume and the possibility of continuously monitoring the density of a fluid with high resolution.

In this paper, a straight silicon tube, a fixed-fixed resonating structure, is fabricated and evaluated as a density sensor (see fig. 1). The absence of corners leads to less flow restriction and a reduced risk of trapping gas bubbles and micro-particles in the sensor. In this design, the sample volume is just $9.3 \mu \mathrm{L}$. The sensor consists of a $2.65 \mathrm{~cm}$ long resonating tube mechanically supported by the surrounding silicon frame. Electrodes were patterned on an etched recess of a glass slide with a depth of $6 \mu \mathrm{m}$. An aluminum fixture clamps the slide beneath the resonating element and also connects an external tube system to the inlet/outlet of the tube.

In this work, the fabricated sensor was calibrated as a density sensor using different known fluids and the Q-factor and the density sensitivity, two important parameters of a densitometer, are measured for its first three vibration modes.

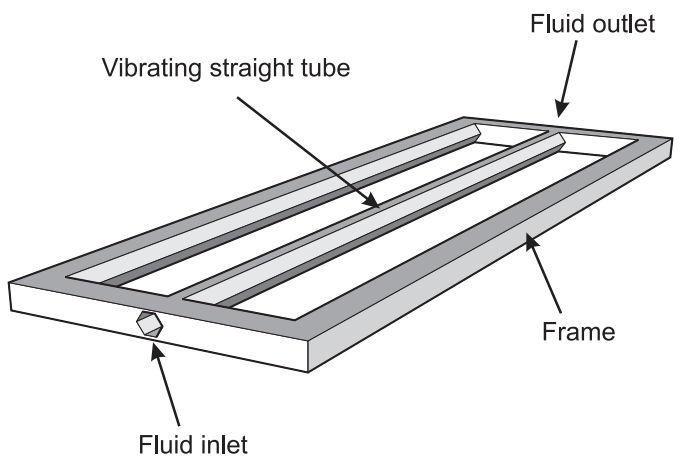

Fig. 1. Straight tube densitometer

\section{THEORY}

By using an earlier derived formula in [12], rewriting it and simplifying all the geometric parameters into one constant $C_{i}$, a formula to calculate the resonance frequency of a clampedclamped tube structure can be provided:

$$
f_{i}=\frac{\lambda_{i}^{2}}{2 \pi L^{2}} \sqrt{\frac{E I}{m}} \Rightarrow f_{i}=C_{i} \sqrt{\frac{E}{\rho_{t} A_{t}+\rho_{f} A_{f}}}
$$

Where $E$ is Young's modulus of $\mathrm{Si}, I$ is the area moment of inertia, $L$ is the length of the tube, $m$ is the total mass of the resonating element (tube and fluid) per unit length and $\lambda_{i}$ is a constant related to the mode of vibration $\left(\lambda_{1}=4.730\right.$, $\lambda_{2}=7.853$ and $\left.\lambda_{3}=10.996\right) . C_{i}$ depends on the tube dimensions and the vibration mode. $\rho_{t}$ and $\rho_{f}$ are the tube and fluid densities, and $A_{t}$ and $A_{f}$ are the cross-sectional areas of the tube walls and the fluid, respectively.

The fluid density sensitivity of mode $i$ of this densitometer can be calculated by:

$$
S_{i} \equiv \frac{1}{f_{i}} \frac{\partial f_{i}}{\partial \rho_{f}}=-\frac{1}{2\left(\rho_{f}+\frac{A_{t}}{A_{f}} \rho_{t}\right)}
$$

The $A_{t} / A_{f}$ design ratio is 0.535 yielding a theoretical fluid density sensitivity of $-223 \mathrm{ppm} / \mathrm{kg} \cdot \mathrm{m}^{-3}$ at the water's density for each vibration mode (at room temperature and $1 \mathrm{~atm}$ pressure, $\rho_{t}=2329$ and $\rho_{f}=997 \mathrm{~kg} / \mathrm{m}^{3}$, respectively [13]). 


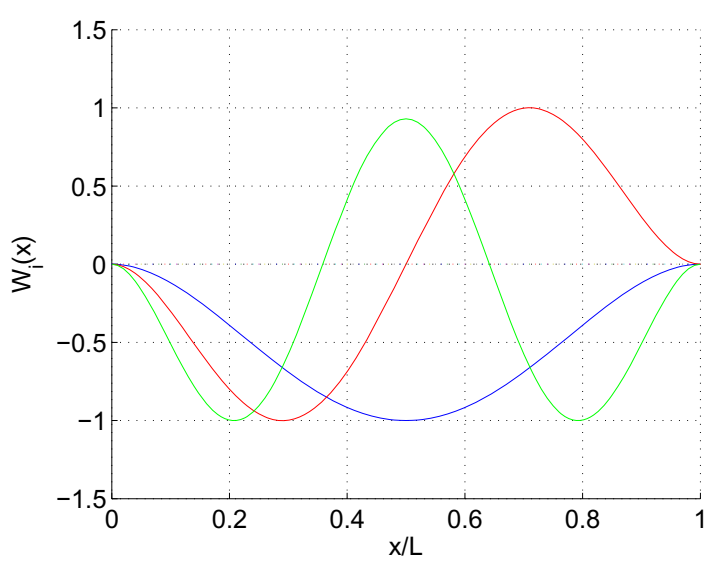

Fig. 2. The first three vibration modes of a fixed-fixed resonating tube. $L$ is the length of the tube and $W_{i}(x)$ is the normalized deflection.

\section{DESIGN AND FABRICATION}

The main steps of the fabrication process were $\mathrm{KOH}$ etching and silicon fusion bonding. $75 \mathrm{~mm}$ (100)-silicon wafers were thermally oxidized to produce $300 \mathrm{~nm}$ oxide. LPCVD was used to deposit $100 \mathrm{~nm}$ nitride. Two photolithography and RIE steps were used to create a nitride mask on the inside side and a nitride pattern on the outside side of the wafers. Then, $\mathrm{KOH}$ etching was used to create the interior of the tubes. After stripping the nitride and oxide on the inside side of two wafers by an RIE and HF etch, they were bonded using silicon fusion bonding.

After bonding, $\mathrm{KOH}$ was used to etch the exterior of tubes to release the structures. Finally the nitride/oxide mask was stripped using Buffered HF and the wafer was diced to create $10 \mathrm{~mm} \times 28.5 \mathrm{~mm}$ sensor structures.

The diced sensor was fixed to the fixture using an epoxy glue. The glass slide containing the electrodes was fixed beneath the tube by the fixture.

Fig. 4 shows the dimensions of the sensor and the crosssection of the glued sensor in the fixture.

\section{EVALUATION RESULTS}

The diced silicon sensor was glued to an aluminum fixture using an epoxy glue. Then, a conductive glue was used to create an ohmic-contact between the sensor and the fixture. Steel tubes were used to lead the fluid to the tube. The electrodes were sputtered through a shadow mask in an etched recess of a glass slide. The slide was placed and fixed beneath the tube by the fixture. The electrodes were parallel to the tube and because of chip bow, the distance between the middle electrode and the tube was smaller than the nominal gap of $6 \mu \mathrm{m}$. The bow direction was used to assist exciting the tube with a lower amplitude voltage.

The sensor was placed at a vacuum chamber and a laser Doppler vibrometer was used to detect and measure the first three resonance frequencies with $1 \mathrm{~Hz}$ resolution. Air and five different 2-propanol/water mixtures were used to calibrate

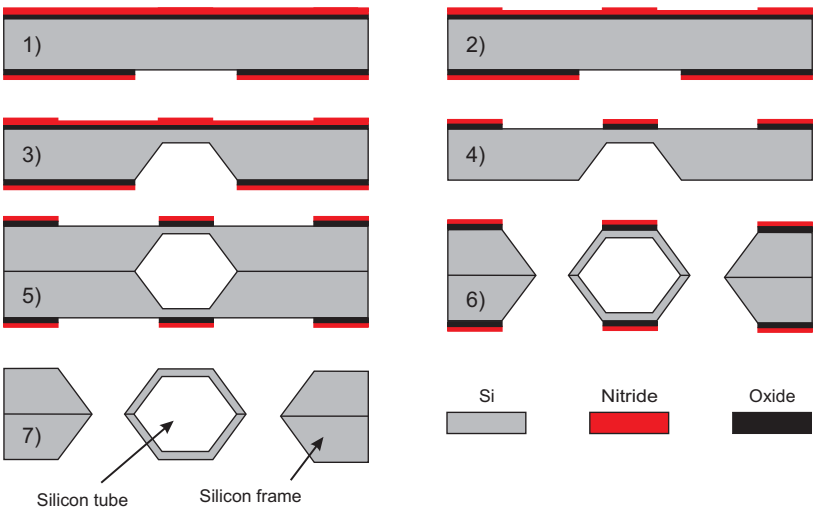

Fig. 3. Process steps: 1) photolithography and RIE etch of the bonding side (inside side); 2) photolithography and RIE etch of the outside side; 3) $\mathrm{KOH}$ etch to create the interior of the tubes; 4) partially etch the nitride from the outside side, etch all nitride from the bonding side, etch oxide on both sides and prepare for bonding using Piranha; 5) silicon-silicon fusion bonding of a pair of wafers; 6) $\mathrm{KOH}$ etch to create the exterior of the tubes; 7) strip the oxide/nirtride mask using buffered oxide HF (BOE) and dice the structures

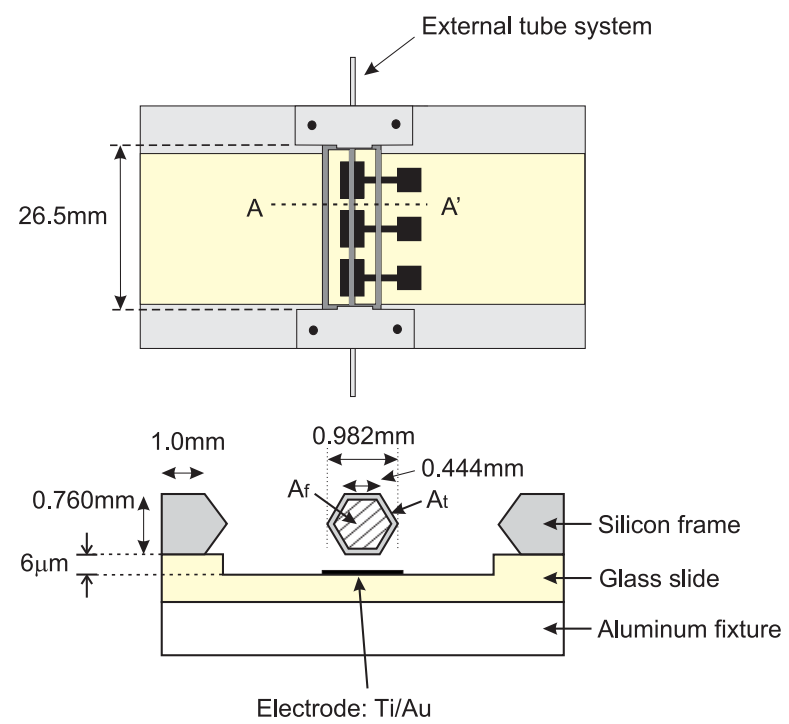

Fig. 4. The density sensor in its fixture (top); Cross section A-A' of the sensor and fixture with the design dimensions (bottom)

the density sensor. The density of each liquid mixture was measured using a scale and a volumetric flask. Table I shows the evaluation results at $2 \times 10^{-4}$ mbar chamber pressure. $1 / f_{i}^{2}$ is plotted versus density in fig. 5. Three regression lines are passed through the points of each mode using least square approximation to produce the calibration curves. Fig. 6 plots the first resonance frequency versus fluid density.

\section{DiscusSiON}

\section{A. Quality values}

The observed Q-values for air and two liquid fluids at $2 \times$ $10^{-4}$ mbar chamber pressure are presented in table II. The fact that the Q-value is larger for the higher modes can be explained by the induced vibrations to the frame. The center of gravity of the vibrating element moves up and down in mode 1 causing 


\begin{tabular}{c|c|c|c|c|c}
$x_{V}(\mathrm{~W})$ & $x_{V}(\mathrm{IPA})$ & $\rho\left(\mathrm{kg} / \mathrm{m}^{3}\right)$ & $f_{1}(\mathrm{~Hz})$ & $f_{2}(H z)$ & $f_{3}(H z)$ \\
\hline 1.00 & 0.00 & 997.70 & 7127 & 19661 & 38125 \\
0.75 & 0.25 & 958.92 & 7176 & 19822 & 38452 \\
0.50 & 0.50 & 912.19 & 7254 & 20027 & 38800 \\
0.25 & 0.75 & 853.70 & 7381 & 20337 & 39370 \\
0.00 & 1.00 & 779.11 & 7567 & 20847 & 40440 \\
Air & - & 1.2047 & 10104 & 28402 & 54100
\end{tabular}

$x_{V}(\mathrm{~W})$ : approximate volumetric fraction of water in the mixture.

$x_{V}$ (IPA): approximate volumetric fraction of 2-propanol in the mixture.

TABLE I

$\rho_{f}$ AND $f_{i}$ MEASUREMENTS AT $2 \times 10^{-4}$ mbar CHAMBER PRESSURE

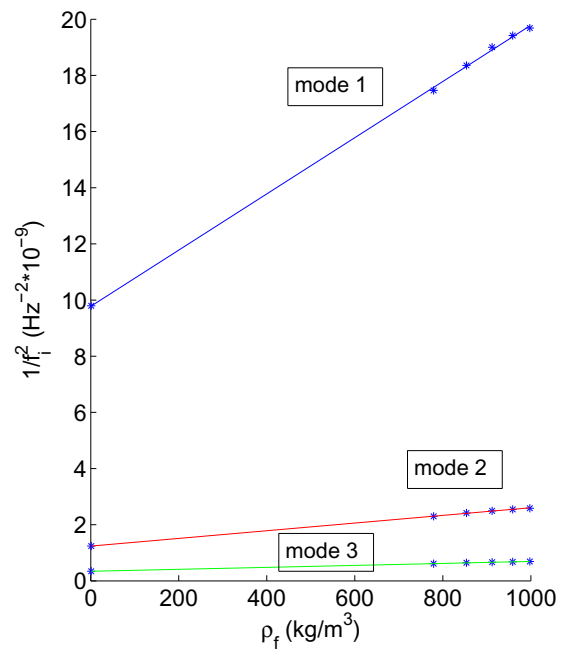

Fig. 5. $1 / f_{i}^{2}$ plot of six fluids and the relevant regression lines using leastsquare approximation.

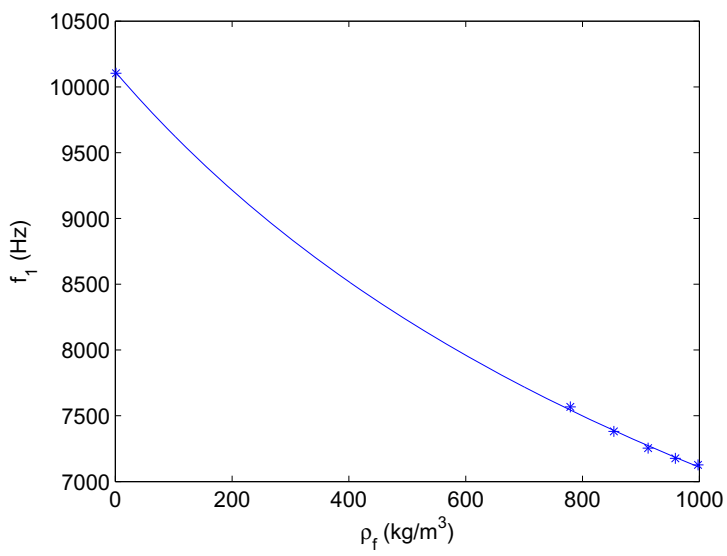

Fig. 6. Plot of $f_{1}$ vs. fluid density for six fluids and curve through the measured points using the regression line parameters derived from fig. 5

\begin{tabular}{c|c|c|c} 
fluid & $\mathrm{Q}_{1}$ & $\mathrm{Q}_{2}$ & $\mathrm{Q}_{3}$ \\
\hline water & 61 & 72 & 97 \\
2-propanol & 54 & 70 & 93 \\
gas* & 138 & 157 & 244
\end{tabular}

*: the pressure inside the tube equals the chamber pressure.

TABLE II

The MEASURED Q-VAlues AT $2 \times 10^{-4}$ mbar CHAMber PRESSURE

\begin{tabular}{l|c|c|c} 
& $A_{i} / A_{j}$ & $B_{i} / B_{j}$ & $\left(\lambda_{j} / \lambda_{i}\right)^{4}$ \\
\hline $\mathrm{i}=1 ; \mathrm{j}=2$ & 7.882 & 7.338 & 7.599 \\
$\mathrm{i}=1 ; \mathrm{j}=3$ & 28.63 & 28.56 & 29.20 \\
$\mathrm{i}=2 ; \mathrm{j}=3$ & 3.632 & 3.893 & 3.843
\end{tabular}

TABLE III

THe PRACTICAL AND theORETICAL $A_{i} / A_{j}$ AND $B_{i} / B_{j}$ VAlues

the frame to vibrate. Momentum instability in mode 2 causes the frame to vibrate transversely. In mode 3 , the center of mass of the vibrating tube goes up and down but this effect is less than mode 1 . These descriptions with observed a stiffer frame at higher frequencies can describe increasing the Q-value by mode number. The liquid-filled tubes have also shown a lower Q-value because of possible internal liquid damping.

The authors speculate that in the case of encapsulating the sensor, the loss through the frame and the glued parts, generally called clamping loss, could be eliminated leading to have a higher Q-value.

At room temperature, the viscosity of 2-propanol is more than twice that of water [11]. The measured Q-factors of the water and 2-propanol filled tubes lay within the error margins of the used measurement method. This observation, together with the observed linear calibration curves, lead the authors to conclude that the un-encapsulated sensor with this fixture design measures density almost independently of viscosity. Using a more viscous liquid such as glycerol and also improving the Q-value by encapsulating the device to reduce clamping losses can help to show the effect of viscosity of the characteristics of the sensor.

\section{B. Statistical calculations}

The equation of the regression line corresponding the vibration mode $i$ in fig. 5 can be represented as $1 / f_{i}^{2}=B_{i} \cdot \rho_{f}+A_{i}$, where $B_{i}$ and $A_{i}$ are the slope and the y-intercept of the ith regression line, respectively. By putting this equation in equation 1 , it can be shown that for modes $i$ and $j$, the $B_{i} / B_{j}$ and $A_{i} / A_{j}$ fractions are equal to $\left(\lambda_{j} / \lambda_{i}\right)^{4}$ in theory. Table III shows that the practical values of $B_{i} / B_{j}$ and $A_{i} / A_{j}$ for the first three modes are close to their theoretical values validating the theory behind the sensor.

The fluid density sensitivity of each mode at the density of water is presented in table IV. The average observed sensitivity equals $-256 \pm 6 \mathrm{ppm} / \mathrm{kg} \cdot \mathrm{m}^{-3}$ for water. Fig. 7 represents the fluid density sensitivity of each mode as a function of density.

By comparing the regression line equation with equation 1 it can be shown that for mode $i$, the actual $A_{t} / A_{f}$ ratio of 


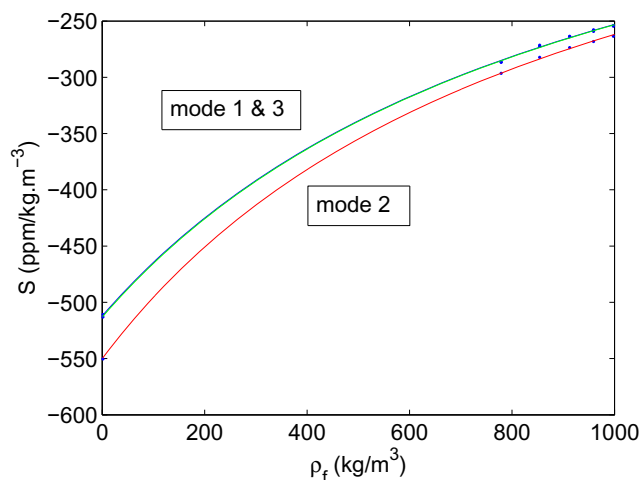

Fig. 7. Plot of fluid density sensitivity vs. fluid density for six fluids. The solid lines are the derivatives of the regression lines in fig. 5 .

\begin{tabular}{c|c|c} 
mode number & $\mathrm{S}_{i}\left(\mathrm{ppm} / \mathrm{kg} \cdot \mathrm{m}^{-3}\right)$ & $A_{i} /\left(B_{i} \cdot \rho_{t}\right)$ \\
\hline $\mathrm{i}=1$ & -253 & 0.419 \\
$\mathrm{i}=2$ & -262 & 0.390 \\
$\mathrm{i}=3$ & -254 & 0.418
\end{tabular}

TABLE IV

The observed $S_{i}$ And $A_{t} / A_{f}$ VAlues for three Vibration modes

the tube equals $A_{i} /\left(B_{i} \cdot \rho_{t}\right)$. The actual $A_{t} / A_{f}$ ratios and the fluid density sensitivity of each mode for water density are presented in table IV. The average observed area ratio and sensitivity for water are 0.409 and $-256 \pm 6 \mathrm{ppm} / \mathrm{kg} \cdot \mathrm{m}^{-3}$, respectively. Alignment inaccuracies in the four photolithography steps and in the bonding step, mask underetch during the fabrication process and the inaccuracy in the thickness of the wafers result in a smaller $A_{t} / A_{f}$ ratio in practice.

The $A_{t} / A_{f}$ value measured from a microscope cross section of a tube fabricated by similar process (see fig. 8) was found to be approximately 0.386 , which according to the model would result in a sensitivity of $-264 \mathrm{ppm} / \mathrm{kg} \cdot \mathrm{m}^{-3}$ for water. This calculated sensitivity lies close to the average measured sensitivity of $-256 \mathrm{ppm} / \mathrm{kg} \cdot \mathrm{m}^{-3}$ for water, confirming the validity of the model.

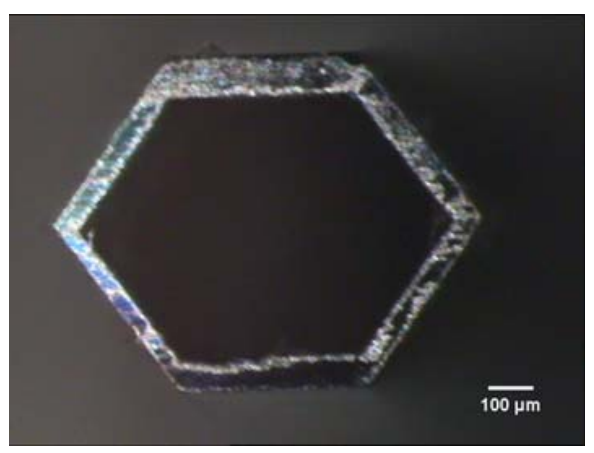

Fig. 8. Cross section of a fabricated tube

\section{CONCLUSION}

A straight tube has been fabricated and evaluated as a density sensor. Its first three vibration modes were calibrated using air and different liquid mixtures. The three modes have shown an average fluid density sensitivity of $-256 \pm 6 \mathrm{ppm} / \mathrm{kg} \cdot \mathrm{m}^{-3}$ around the density of water which is proportional to the area ratio of the tube and the fluid in theory. By passing an unknown fluid through the sensor and by measuring the resonance frequencies and using the calibration curves, the density can be extracted.

The sensor should be vacuum encapsulated to eliminate the structure loss and clamping loss to have a higher Q-value.

\section{ACKNOWLEDGMENT}

The authors thank Henri Jansen, University of Twente in the Netherlands, for his advice on direct wafer bonding.

\section{REFERENCES}

[1] P. Enoksson, G. Stemme and E. Stemme, "Silicon tube structures for a fluid density sensor", Sensors and Actuators A: Physical, Vol. 54, Issues 1-3, June 1996, pp. 558-562

[2] D. Sparks, R. Smith, M. Straayer, J. Cripe, R. Schneider, A. Chimbayo, S. Ansari and N. Najafi, "Measurement of density and chemical concentration using a microfluidic chip," Lab Chip, Vo1. 3, pp. 19-21 (2003).

[3] D. Westberg, O. Paul, G. I. Andersson and H. Baltes, "A CMOScompatible device for fluid density measurements", Micro Electro Mechanical systems, 1997. MEMS' 97, Proceedings, IEEE, 10th Annual International Workshop on 26-30 Jan 1997, pp. 278-283.

[4] D. Sparks, V. Cruz and N. Najafi, "The resonant behavior of silicon tubes under two-phase microfluidic conditions with both microbeads and gas bubbles", Sensors and Actuators A 135, 2007, Pages 827-832.

[5] W. K. Schomburg, M. Vitt, W. Bacher, M. W. Borner and W. Menz, "Measurements of physical parameters with ultrasound and microdiaphragms", Micro Electro Mechanical Systems, 1995, MEMS '95, Proceedings, IEEE, 29 Jan.-2 Feb. 1995 Pages: 139-144.

[6] W. Y. Shih, X. Li, H. Gu, W. H. Shih and I. A. Aksay, "Simultaneous liquid viscosity and density determination with piezoelectric unimorph cantilevers", J. Appl. Phys. 89, 1497 (2001).

[7] C. Harrison, S. Ryu, A. Goodwin, K. Hsu, E. Donzier, F. Marty and B. Mercier, "A MEMS sensor for the measurement of density-viscosity for oilfield applications", Proc. SPIE Int. Soc. Opt. Eng. 6111, 61110D (2006).

[8] B. A. Martin, S. W. Wenzel and R. M. White, "Viscosity and Density Sensing with Ultrasonic Plate Waves", Sensors and Actuators A: Physical, Volume 22, Issues 1-3, June 1989, Pages 704-708.

[9] J. Zhang, C. Dai, X. Su and S. J. O'Shea, "Determination of liquid density with a low frequency mechanical sensor based on quartz tuning fork", Sensors and Actuators B: Chemical, Volume 84, Issues 2-3, 15 May 2002, Pages 123-128.

[10] C. A. Grimes, D. Kouzoudis and C. Mungle, "Simultaneous measurement of liquid density and viscosity using remote query magnetoelastic sensors", Rev. Sci. Instrum. 71, 3822 (2000).

[11] D. R. Lide and H. V. Kehiaian, "CRC handbook of thermophysical and thermochemical data", CRC, 1994.

[12] R. D. Blevins, "Formulas for Natural Frequency and Mode Shape", Van Nostrand Reinhold Company, 1979.

[13] D. R. Lide, "CRC handbook of chemistry and physics", Ed. 80, CRC, 1999. 